\title{
Perceptions of marijuana use: Chronicles of marijuana smokers from two marijuana-Growing communities in South Africa
}

\author{
Emmanuel Manu ${ }^{1 *}$, Mohlomi Jafta Ntsaba ${ }^{2}$ \\ 1, 2 Walter Sisulu University, Mthatha, South Africa
}

Key Words:

Perceptions

Marijuana

Chronicles

Smokers

South Africa

Received: 08 September 2016 Accepted: 18 September 2016 Published: 25 October 2016

\begin{abstract}
Marijuana use in South Africa has been extensively researched and documented over the last thirty years. Functionalist theorists posit that societal norms and practices, which are passed on from generation to generation in a top-down approach, sometimes play a role in influencing individuals to use illicit substances such as marijuana. Perceptions of marijuana in a community are likely to be passed on to adolescents from adults, influencing them to indulge in marijuana smoking. However, the perceptions that marijuana smokers hold of the drug, especially in marijuana-growing communities, have not received the needed academic attention. This article therefore seeks to shed light on the perceptions of marijuana smokers in rural marijuana-growing communities in South Africa. Qualitative research methods, underpinned by constructivist philosophical assumptions, were used in this study. Face-to-face semi-structured interview questions were used to collect the data. A total of 17 marijuana smokers, comprising 15 focus-group participants and two key informants, were interviewed in two communities. Two-staged, non-randomized sampling techniques, both purposive and snowballing, were used to select the communities and the individual participants for the study. Purposive sampling was used to select the communities, while the snowball sampling technique was used to select the interviewees. This was done by making contact with a few individuals who smoked marijuana in both communities, who helped recruit the rest of the participants. The thematic content analysis procedure was used to analyse the data for the study. The results obtained revealed that marijuana smokers in the sampled communities hold the belief that marijuana is not harmful. The only negative effect they associate with marijuana use is a social one; that after using the substance, they are perceived by others as being disrespectful and lazy. It was also established that most users smoke marijuana for the perceived spiritual and medical benefits that they associate with marijuana use. Female marijuana smoking was uniformly frowned upon by all participants in the study, who regard such users as social outcasts. Perceptions toward marijuana use among the youth in marijuana-growing communities need serious attention if the prevalence of marijuana use in such communities is to be stemmed. Intervention programmes tailored for rural marijuana-growing communities need to be designed to deal with the complex social and economic factors that uphold marijuana's position in such communities.
\end{abstract}

(C) 2017 The Author(s). Published by TAF Publishing.

\section{INTRODUCTION}

Marijuana is one of the most widely-used illicit substances in the world, and often serves as the drug of choice for adolescents before their initiation into the use of other illicit substances such as cocaine [4]. It is estimated that there are about 18.1 million current marijuana users in the United States [18], [23]. The National Institute on Drug Abuse [18] attributed the high rate of marijuana use in the U.S. to the availability and ease of access

\footnotetext{
${ }^{*}$ Corresponding author: Emmanuel Manu

${ }^{\dagger}$ Email: emanu338@gmail.com
} 
to the drug in the country. Also, shifting perceptions towards marijuana use have been found to be a key factor in maintaining its high rate of usage in the U.S. According to The National Institute on Drug Abuse [18] most North American youth believe that marijuana is not harmful.

In most European countries, marijuana use is prevalent among the youth. A research report published by the European Commission [9] revealed that at least $6 \%$ of youth in European countries used the drug. The high prevalence of marijuana use in most European Union countries has been attributed to a lack of uniform and coherent policies on illicit drug use among member countries [6]. For instance, while Sweden has adopted a moralistic stance on drug use and aims to eradicate the use of illicit drugs altogether, the Netherlands has adopted a pragmatic stance, with the aim of merely minimizing the harm caused by illicit drugs [6]. Irrespective of the nature of drug policy a country adopts, the European Commission [9] maintained that individual perceptions towards marijuana primarily determine its use in the region. The Commission explained that most European youth believed that using marijuana once or twice a week posed a low to no health risk, with Spanish, Slovakian and Czech Republic youth being the heaviest users.

African youth are not insulated from global perceptions of marijuana. According to Shehu et al. [25], marijuana use among adolescents in Zaria, Nigeria, was as high as $9.4 \%$. This figure includes both able-bodied users and those with disabilities. Oyewumi et al. [19] found high marijuana use among adolescents with hearing impairments in Nigeria, despite the high level of awareness that the majority, including those with disabilities, had of its health risks. Marijuana use has gained popularity in South Africa over the years. South African Christian Addiction Support [7], an organisation that assists in the rehabilitation of drug addicts, reported that marijuana use in the country increased by $20 \%$ in the two years from 2011 to 2012. In 2013, the number of people admitted to treatment centres across the country for marijuana dependency increased by a staggering $22 \%$ [27].

While marijuana usage is a national problem in South Africa, its cultivation and production is confined to specific areas. According to the United Nations Office on Drug and Crime [31], illegal commercial marijuana production in South Africa is concentrated on the coastal belts of KwaZulu Natal and the Eastern Cape Provinces. In the Eastern Cape, the plant is extensively cultivated in the Pondoland region of the Wild Coast [14], and has been for many years. Traditionally, marijuana use in these marijuana-growing communities was regulated by strict rules enacted by traditional leaders. The grip those leaders had on their communities has eroded due to modernisation, leading to the widespread and uncontrolled smoking of marijuana in such communities [20].

Despite an increase in knowledge regarding marijuana use in South Africa, little is known about the perceptions that marijuana users themselves have of the drug, particularly in areas where its production occupies a central economic role. This paper therefore explores the perceptions that marijuana smokers in two marijuana-growing communities in the Eastern Cape Province of South Africa have of marijuana, in the hope of contributing to the body of knowledge regarding this powerful and widely-accepted drug.

\section{THEORATICAL PERSPECTIVE OF THE STUDY}

The study was underpinned by the functionalist theory, often employed in sociology. According to Livesey [16], functionalist sociology views society as being composed of two major systems; the social system and the social structure. The social system is an organised structure of interrelated parts called institutions. The social structure, on the other hand, refers to specific ways in which various institutions are related to one another on 
a functional basis. Social system and social structure together form what is referred to as the social institution. The social institution, according to Livesey [16], is a large scale, organised behaviour pattern that exists over a long period of time. People are born into these already existing systems of institutional arrangements which play a significant role in relation to their culture [22].

Spencer-Oatey [2] defines culture as the broad patterns of values and beliefs that characterise a way of life and which are transmitted from generation to generation in a particular society. Individuals are born into a society and become the products of all the social influences around them, as they are socialised by various societal institutions such as family, friends, education, media and religion [22]. Societal norms, beliefs and perceptions of behaviour, such as marijuana smoking, therefore transfer easily from one generation to another. Härkönen [11] reckoned that the family and peers are immediate community institutions, and play the strongest role in inculcating societal norms in an individual. An individual would be more likely to accept and begin marijuana smoking if the people by whom he or she is surrounded indulge in the behaviour. Moreover, one is likely to uphold the views shared by one's society without verifying the veracity of such views if alternative, more accurate views are not commonly espoused.

\section{The Purpose of the Study}

Reflecting on the topic and the theoretical background, this study sought to explore the perceptions that marijuana smokers held towards marijuana in two marijuana-growing communities in the Pondoland region of South Africa.

\section{RESEARCH METHODOLOGY}

Descriptive phenomenological qualitative research design was employed in this study, as the study aimed at providing a textual description of participants' beliefs and perceptions about marijuana use [21]. Thus, the study was concerned with developing explanations of a social phenomenon, with the aim of understanding the world in which they occur. Descriptive qualitative methods helped to achieve the aim of the study by shedding light on participants' belief systems. Moreover, the qualitative methodology allowed the researcher to use a relatively small but information-rich sample to establish knowledge on the research problem instead of using a large number of participants which is a typical characteristic of quantitative or mixed methodology studies [12].

\section{Sample Population}

A total of 17 participants from two communities, "Khanya" community and "Ilanga" community (assumed names), were non-randomly selected for the study. One focus group per community was formed, each comprising male marijuana smokers aged 17-25 years. In all, there were 15 marijuana-smoking focus group members from both communities. In each community, one adult marijuana-smoking key informant aged over 40 years per community was also selected to share his experiences and views on the topic. There was no female participant in the study. This was because female marijuana smokers were not known in either of the communities and could therefore not be recruited. The focus group participants were aged from 17 years to 25 years, while the marijuana smoking key informants were all aged above 25 years. None of the participants had attained a tertiary education. A two-staged non-probability sampling procedure was followed to select the communities and the individual participants for the study. These included, firstly, purposive sampling and secondly, the snowball sampling technique [13]. The purposive sampling tech- 
nique was used to select the communities where the research was conducted. Given that the study investigated perceptions towards marijuana use in marijuana-growing communities, it was imperative to select communities where marijuana was known to be cultivated - hence the Pondoland region the Eastern Cape.

Secondly, the snowball technique was used to select individual participants for the study. It involved the assistance of study participants in recruiting marijuana smokers, as it was difficult for the researcher to gain access to such a population [5]. The process started with the identification of key informants who were marijuana smokers and lived in the two communities where the study was conducted. The key informants helped the researcher to identify and recruit more marijuana smokers to form focus groups who were then interviewed.

\section{Data Collection}

The data were collected using a face-to-face semi-structured interview guide. The interview guide was written in both English and IsiXhosa. It was first compiled in English, then translated to IsiXhosa and back into English by a professional translator to ensure that the meaning of the questions was not lost during the translation process. A pilot study was then conducted in a community with similar characteristics as the two communities where the actual study was conducted. This was to establish the appropriateness of the interview questions in eliciting appropriate answers and to test participant recruitment procedures [5]. Also, the pilot study served the purpose of testing the overall research design, including the data analysis procedures that were to be employed in the main study [1]. The data collection for the actual study ran from 4 December 2015 to 31 January 2016. The data were collected through interviews with individuals in the focus groups and the key adult informants, with each session lasting about an hour. The interviews were tape recorded with the permission of the participants.

\section{Ethical Considerations}

The research proposal was presented to the Health Sciences Higher Degrees Committee of Walter Sisulu University for scientific and ethical review, and inputs from the committee were then incorporated into the study. The proposal was then submitted to the University Ethics Committee for ethical clearance and for the issuance of an ethics clearance certificate, which was granted. The proposal, with an accompanying permission letter, was then submitted to the chiefs of the various communities to seek permission to conduct the study in their communities. Permission was also sought from community members through community forums. To protect the identity of the communities that participated in the study, the communities were given coded names; Khanya community and Ilanga community. Thus the names are not the actual names of the communities that participated in the study. Individual participants' names were also not revealed during the data collection process or in the compilation of the manuscript to ensure anonymity. Permission to participate for those below the age of 18 was sought from participants' parents as well as the participants themselves. Participants who were permitted by their parents were provided with a full explanation of the study and informed about their rights. With those over 18, the study was explained to them, their rights were explained to them, and their permission was sought to conduct anonymous interviews. Participation was therefore voluntary, with the opportunity to withdraw from the study whenever an interviewee wished to do so. 


\section{Data Analysis}

The data analysis approach adopted in this study was content-based. Vaismoradi et al. [33] explain content analysis as a set of techniques used to analyse textual data and elucidate themes. The data analysis procedure for this study took two forms: First, a qualified language translator transcribed the interview recordings by translating them from IsiXhosa to English.

The researcher then read through them thoroughly and highlighted the themes and sub-themes for easy identification and interpretation. Recurring themes and sub-themes were identified and colour-coded for each of the data categories. This enabled the researcher to determine the emerging themes from the data for easy analysis and interpretation. The second stage involved identifying common themes and areas of variance among the participants, and those themes that emerged were synthesized for appropriate analysis and interpretation [26]. The themes were then used to structure the presentation of the findings provided in this article.

\section{Trustworthiness}

Trustworthiness in qualitative data is the extent to which the research findings are credible and can therefore be defended when challenged [3]. To ensure that the findings of this study were credible, various strategies proposed by Creswell [8] were adhered to. A rigorous methodology was used, comprising the conduction of a pilot study, peer debriefing, member checking, accurate reporting on participants' viewpoint and the use of multiple data sources. All of these helped to ensure that the findings were credible [8].

\section{FINDINGS}

The following key findings emerged from the study:

- Firstly, marijuana smokers consistently denied any negative health consequences associated with marijuana use. There was the perception that smoking marijuana has no negative health consequences.

- Secondly, marijuana smokers believed that marijuana smoking affected only social relationships, in that they were perceived to be lazy and disrespectful after smoking marijuana — and indeed, were so, as acknowledged by them.

- Thirdly, marijuana smokers believed that the plant had positive health benefits to the user. They believed it was capable of treating a host of illnesses such as cough and high blood pressure.

- Fourthly, these marijuana smokers believed that marijuana smoke had strong spiritual properties; it could repel evil spirits responsible for causing nightmares. For this reason, they either smoked the drug before bedtime or burned it in their rooms while sleeping.

- Lastly, female marijuana smoking was perceived to be socially unacceptable behaviour in these marijuana-growing communities, and was hardly known.

\section{DISCUSSION OF FINDINGS}

\section{Denial of Negative Health Consequences of Marijuana Use}

The findings revealed that most marijuana smokers believed that marijuana caused no harm to the user. They claimed to have known people who had smoked marijuana for many years and had suffered no ill consequences; therefore, in their view, marijuana could not be said to cause harm to the smoker. A focus group member from Khanya community said: 
"No, there is no disease that can be caused by it [marijuana] because there are many old people who are smoking it ever since I was born and I have never heard that they are sick because of it or someone has died from smoking it."

It could be deduced from the findings that marijuana-related illnesses or deaths are not reported in the community, although certain illnesses or deaths might have occurred in these communities as a result of marijuana use. There is clearly a myth among marijuana smokers that marijuana is a harmless drug. The myth that marijuana poses no threat to one's health seems to be global. According to Koroma [15], adolescents in Sierra Leone saw marijuana as having no adverse health effects; they even brewed its leaves for drinking. The perception that marijuana is harmless could stem from the fact that most African and South African youth are not well-informed regarding what marijuana does to the human brain and body. According to Mafubelu [17], drug awareness programmes are not as intense in rural parts of South Africa as they should be. This lack of drug awareness programmes in most rural communities, including those where marijuana is grown, has perpetuated the perception that there are no health dangers associated with marijuana smoking. This perception seems to cut across all age groups; the marijuana-smoking key informant in each community, both of whom were much older, had similar perceptions. According to Rakhi et al. [22], transmission of cultural norms is a top-down process. They are passed on from one generation to the next. Hence marijuana smokers in rural communities who believe the drug is harmless have been told by older marijuana smokers that it is harmless, and no awareness programmes have brought about a change in this view. To eradicate this misconception, there is the need to increase public health awareness in the rural communities where marijuana is cultivated. This would increase their knowledge on marijuana's effects and possibly prevent a new generation from following unquestioningly in their elders' footprints.

\section{Perceived Social Problems Associated with Marijuana Use}

Another important finding was that marijuana smokers believed that the problems associated with marijuana use were purely social ones. The smokers explained that after smoking they became lazy and disrespectful, and that this affected their social relationships. They were fully aware of its effects on their behaviour. As a result of their laziness and disrespectfulness, they were not able to perform any household tasks after smoking marijuana, leading them to refuse requests and orders from parents or elder siblings. This lack of a positive response to the order of an older person is taboo in traditional African communities. It deviates completely from the social norm and has a particularly disruptive effect in rural communities, which depend on harmony and cooperation in order to function normally. One smoker explained:

"It makes me disrespectful; I don't care whether the person is old enough to be my mother or my father, I just talk any way I like. I don't feel shy about anything. If someone older than me sends me to do something, I will just say aloud that I am not going to do that. It also makes me lazy. After smoking it, I don't want to do anything".

Adolescents who are shy and are unable to do certain things when sober turn to marijuana to give them the needed courage and conquer their shyness. Marijuana removes the usual social inhibitions and frees them from social conventions, so that they act in ways they never would while sober. While marijuana use is often associated with crime and violence Morris et al. [24] smokers of the drug in Khanya and Ilanga community were insistent that it made them only lazy and disrespectful, never violent. However, laziness and disrespectfulness are both behaviours that are not acceptable in most African cultures. Ac- 
cording to Wallace [34] , the way people behave towards others and the way we engage with others in a relationship are true conveyors of our values; they are attributes we learn in our homes or communities. Wallace [34] posits that being respectful is a prerequisite for successful interaction with others. Being frequently disrespectful and refusing to contribute to household tasks make interaction with people much more difficult and have long-term effects on social relations. In a traditional African community, where conformity with the social values of respect, courtesy and obedience to elders is highly valued, being lazy and disrespectful marks one as socially deviant; it also stigmatises the whole family and possibly the whole community. Marijuana smokers in these communities are also less likely to be considered when employment opportunities arise as the smokers would be perceived as high-risk.

\section{Perceived Health Benefits Associated with Marijuana Use}

It was further revealed that marijuana smokers held deep-seated beliefs regarding the health benefits of marijuana. The plant is used in many different ways by people in the communities studied, including women and children, who used it to treat various illnesses. They saw marijuana as a medicinal plant, and feared no condemnation for using it therapeutically. A range of conditions believed to respond positively to marijuana include pimples, coughs, asthma, diabetes, high blood pressure and tooth-ache. A key informant narrated:

"People with severe cough cook it and drink the 'amatyatya' [the boiled water] to relieve themselves of the cough".

The plant was mostly boiled and the water extracted and drunk as a tea. People suffering from pimples on the face covered their heads with a towel and bent over a bowl of the leaves steeped in boiling water; the steam is believed to be beneficial for skin, possibly through killing the bacteria responsible for the condition. The same procedure was followed by people who suffered from cough symptoms. Although the use of marijuana for treating chronic pain is well documented Zvolensky et al. [35 its use in treating coughs and high blood pressure is not in keeping with the literature. It was explained that a person suffering from high blood pressure boils the drug and drinks the water in the form of marijuana tea over a sustained period of time, which significantly lowers the high blood pressure.

However, Greydanus et al. [10] explain that marijuana increases both heart rate and blood pressure. As a result, it can lead to cannabis arthritis, cardiomyopathy, myocardial infarction, sudden cardiac death, Transient Ischemic Attack (TIA), cerebrovascular accident (stroke), and cardiac arrhythmias. It is therefore evident that participants were ill-informed, not only on the deleterious effects of smoking marijuana, but of its effects on specific conditions when used medicinally without scientific proof. While some used it with the aim of lowering high blood pressure, they were, in fact, increasing their chances of suffering a cardiac arrest.

\section{Perceived Spiritual Attributes of Marijuana Smoke}

It was established that some marijuana smokers believed that marijuana smoke was capable of repelling evil spirits. A smoker explained that marijuana smoke chased away spirits that caused nightmares; they therefore smoked or burned marijuana in their rooms to keep bad spirits away. He claimed:

"...I smoke dagga [marijuana] because I used to have nightmares a lot, so my uncle advised me to smoke it when I am about to sleep or burn it in the room. It chases bad spirits 
away and I no longer have nightmares since I started smoking and burning dagga in my room".

Marijuana is smoked or burned in rooms at night with the aim of preventing nightmares. The belief therefore was that marijuana smoke contained spiritual properties. According to the World Health Organization [30], traditional or spiritual healing is based on beliefs and experiences indigenous to different cultures. In a traditional African community such as these in Pondoland where marijuana cultivation is a cultural norm, there is therefore a high chance that the plant would be imbued with certain perceived qualities other than recreational ones. The perceived spiritual properties of marijuana are transferred from one generation to the next, so that even before an individual contemplates smoking it, he or she has already formed a favourable impression of it from the spiritual beliefs of the community. According to Asamoah-Gyadu [2], belief in supernatural forces and witchcraft is prevalent in the worldview of Africans. In an attempt to fend off evil spirits, various media and practices are consulted or performed, based on one's faith. Hence, the belief in the spiritual properties of marijuana in the Pondoland region is not surprising. In South Africa, nearly $80 \%$ of the population consult traditional or spiritual healers to deal with physical and spiritual ailments [29]. Traditional and spiritual healing therefore has a special place in health care delivery in South Africa.

\section{The Unacceptability of Female Marijuana Smoking}

It was established that females in these communities did not customarily smoke marijuana and if they ever did, they did so only in secret. Female marijuana smokers were almost unheard of in these communities. One smoker explained:

"It's mostly we, the boys; the girls don't smoke it [marijuana]. There may be a few of them but it's hard to see a girl smoking in public like we do. It's the useless ones who smoke because their parents have already given up on them because they won't receive "lobola" [bride price] for them. No one will marry a girl who smokes dagga [marijuana]".

Girls who smoked marijuana were regarded as hopeless outcasts in their communities and were considered unlikely to get husbands in the future. The fear of being stereotyped and labelled as a social deviant was a serious concern that prevented women from smoking marijuana, as they did not want to jeopardise their chances of marrying. In these communities, it is a shame for a girl to disappoint her parents by not receiving her bride price in the form of live cattle; therefore males dominate in marijuana use. The phenomenon of male dominance with regard to illicit drug use is a worldwide problem. In most countries across the world, males tend to use drugs more than their female counterparts. The United Nations Office on Drugs and Crime Prevention [32] reckons that more males used marijuana and opiates than females. The problem of male dominance in marijuana use is particularly pronounced in the Pondoland area where marijuana is cultivated. As explained by Rakhi et al. [22], socially structured norms are easily adopted and adhered to by young members of a community. This being the case, girls in Pondoland simply stay away from marijuana, whereas males' use of the drug is tolerated.

\section{CONCLUSION}

Community perceptions of marijuana use in rural communities are transferred from generation to generation and are hence a long-term matter. Marijuana smokers therefore who hold the view that their habit is harmless and indeed beneficial are thus not too far out of line with the prevailing view in these communities, where educational levels are not high and where awareness programmes are scarce. Marijuana smoking or usage in any form 
is not considered bad behaviour by smokers in marijuana-growing communities. Smokers in particular believe that smoking the drug has no negative health effects and indeed benefits them, at least spiritually. There is therefore the need to formulate and implement community-specific drug awareness programmes in communities where marijuana is known to be cultivated. This could aid in increasing knowledge and awareness regarding the dangers associated with marijuana use and help to minimize the use of the drug among a new generation of community members. Moreover, the economic and social factors that lead to the cultivation and ease of access to the drug need to be addressed, so that children are not pre-predisposed to use the drug before they are able to make informed decisions.

\section{REFERENCES}

1. Arain M, Campbell MJ, Cooper CL, Lancaster GA. What is a pilot or feasibility study? A review of current practice and editorial policy. BMC Medical Research Methodology. 2010; 10(1): 67-73. DOI: 10.1186/1471-2288-10-67

2. Asamoah-Gyadu JK. Witchcraft accusations and Christianity in Africa. International Bulletin of Missionary Research. 2015; 39(1): 23-27. DOI: 10.1177/239693931503900107

3. Bashir M, Afzal MT, Azeem M. Reliability and validity of qualitative and operational research paradigm. Pakistan Journal of Statistics and Operation Research. 2008; 4(1): 35-45. DOI: 10.18187/pjsor.v4i1.59

4. Bonn-Miller MO, Harris AH, Trafton JA. Prevalence of cannabis use disorder diagnoses among veterans in 2002, 2008, and 2009. Psychological Services. 2012; 9(4): 404-416. D0I: 10.1037/a0027622

5. Brink H, Van der Walt C, Van Rensburg G. Fundamentals of research methodology for healthcare professionals. Cape Town, CA: Juta \& Company Ltd; 2012.

6. Chatwin C. Mixed messages from Europe on drug policy reform: The cases of Sweden and the Netherlands. Journal of Drug Policy Analysis. 2016. DOI: 10.1515/jdpa-2015-0009

7. Christian Addiction Support. South Africa addiction statistics. 2013. Available from: goo.gl/fuDyxe

8. Creswell JW. Research design: Qualitative, quantitative and mixed methods approaches. London, UK: Sage Publications, Inc; 2009.

9. European Commission. Youth attitudes on drugs: Analytical report. 2011. Available from: goo.gl/06NvSs

10. Greydanus DE, Kaplan G, Baxter LE, Patel DR, Feucht CL. Cannabis: The never-ending, nefarious nepenthe of the 21st century: What should the clinician know? Disease-a-Month. 2015; 61(4): 118-175.

DOI: 10.1016/j.disamonth.2015.01.004

11. Härkönen U. The Bronfenbrenner ecological systems theory of human development. Paper presented at: The 5thInternational Conference, Daugavpils University, Saule, LV; 2007.

12. Hashemnezhad H. Qualitative content analysis research: A review article. Journal of ELT and Applied Linguistics. 2015; 3(1): 2347-6575.

13. Hennink M, Hutter I, Bailey A. Qualitative research methods. London, UK: Sage Publications; 2011.

14. Kepe T. Cannabis sativa and rural livelihoods in South Africa: Politics of cultivation, trade and value in Pondoland. De velopment Southern Africa. 2003; 20(5): 605-615. DOI: 10.1080/0376835032000149252

15. Koroma A. Young people in Sierra Leone today: Challenges, aspirations, experiences. 2012. Available from : goo.gl/HUyE25

16. Livesey C. Functionalist theories: Sociology central notes. 2010. Available from : goo.gl/f6jT3Z

17. Mafubelu D. Substance use, misuse and abuse amongst the youth in Limpopo province. 2013. Available from: goo.gl/ix6lSb

18. National Institute on Drug Abuse. Drug facts: High school and youth trends. 2014. Available from : goo.gl/kYbvbF

19. Oyewumi A, Adigun OT. Determinants of knowledge and attitude towards substance use and abuse among adolescents with hearing impairment in Ibadan, Oyo State. Nigerian School Health Journal. 2014; 25(1): 62-71.

20. Peltzer K, Ramlagan S. Cannabis use trends in South Africa. South African Journal of Psychiatry. 2007; 13(4): 126-131. 
21. Polkinghorne DE. Language and meaning: Data collection in qualitative research. Journal of Counseling Psychology.2005; 52(2): 137-145. DOI: 10.1037/0022-0167.52.2.137

22. Rakhi N, Licy AD, Mohamad NPH. Theoretical perspectives in sociology. Semester course notes. 2011. Available from: goo.gl/rzHyOo

23. Resko SM. Public perceptions and attitudes toward adolescent marijuana use. Sage Open. 2014; 4(1): 1-11.

24. Morris RG, Ten Eyck M, Barnes JC, Kovandzic TV. The effect of medical marijuana laws on crime: Evidence from state panel data, 1990-2006. PloS One. 2014; 9(3): e92816. DOI: 10.1371/journal.pone.0092816

25. Shehu AU, Idris SH. Marijuana smoking among secondary school students in Zaria, Nigeria:Factors responsible and effects on academic performance. Annals of African Medicine. 2008; 7(4): 175-179. DOI: 10.4103/1596-3519.55657

26. Silverman D. Doing qualitative research. London, UK: Sage Publications Ltd; 2013.

27. South African National Council on Alcoholism. National position paper on Cannabis sativa. 2014. Available from: goo.gl/Cxcpef

28. Spencer-Oatey H. What is culture? A compilation of quotations: Global PAD core concepts. 2012. Available from : http://go.warwick.ac.uk/globalpadintercultural

29. The Media Club South Africa. Health care in South Africa. 2012. Available from : goo.gl/CiLXs4

30. The World Health Organization. An overview of the traditional medicine situation in the African Region. 2010. Available from: goo.gl/rZ4w89

31. United Nations Office on Drug and Crime. Drug situation, ODC country profile: South Africa. 2002. Available from: goo.gl/aedkrA

32. United Nations Office on Drugs and Crime Prevention. World drug report. 2014. Available from: goo.gl/FV90Dy

33. Vaismoradi M, Jones J, Turunen H, Snelgrove S. Theme development in qualitative content analysis and thematic analysis. Journal of Nursing Education and Practice. 2016; 6(5): 100-110. D0I: 10.5430/jnep.v6n5p100

34. Wallace M. From principle to practice! A user's guide to do no harm. 2014. Available from: goo.gl/OKUTxS

35. Zvolensky MJ, Cougle JR, Bonn-Miller MO, Norberg MM, Johnson K, Kosiba J, Asmundson GJ. Chronic pain and marijuana use among a nationally representative sample of adults. The American Journal on Addictions. 2011; 20(6): 538-542. DOI: $10.1111 / j .1521-0391.2011 .00176 . x$

— This article does not have any appendix. - 